Compression Molding of Carbon/Polyether ether ketone Composites: Squeeze Flow Behavior of Unidirectional and Randomly-Oriented Strands

Gilles-Philippe Picher-Martel, McGill University

Dr. Arthur Levy, McGill University

Prof. Pascal Hubert, McGill University 


\title{
Compression Molding of Carbon/Polyether ether ketone Composites: Squeeze Flow Behavior of Unidirectional and Randomly-Oriented Strands
}

\author{
Gilles-Philippe Picher-Martel, Arthur Levy and Pascal Hubert ${ }^{1}$ \\ Department of Mechanical Engineering, McGill University, 817 Sherbrooke St. West, Montréal, QC, \\ H3A 0C3, Canada
}

\begin{abstract}
Compression molding of Randomly Oriented Strands (ROS) of thermoplastic composite is a new process that enables the forming of complex shapes with high fiber volume fraction. During compression molding of ROS, several deformation mechanisms occur. This paper focuses on the macroscopic squeeze flow mechanism. It rules how the material will flow and fill intricate features of the mold. The squeeze flow behavior under large strain was investigated for Unidirectional (UD) and ROS. An experimental characterization was performed using an instrumented hot press. Also, to predict the associated thickness reduction, existing models using equivalent viscosity and lubrication assumptions were used. The results showed that large deformation of UD and ROS composite materials is mainly governed by two regimes: a Non-Newtonian fluid behavior at low strain followed by a yielded phase at large strain. Quantitative indicators were defined to analyze these two phases. They showed that current models available in the literature fail to predict accurately the squeeze flow of thermoplastic composites under high strain (>50\%). Also, strands size (especially strand length) has a large effect on the squeeze flow mechanism. This paper provides basic process window guidelines in terms of minimum pressure and achievable strain for compression molding of ROS parts.
\end{abstract}

Keywords : Thermoplastic Composites, Forming Process, Randomly-Oriented Strands, Compression Molding Process, Squeeze Flow

\footnotetext{
${ }^{1}$ Corresponding Author
} 


\section{Introduction}

In the past decades, composite materials have gained a lot of popularity in the automotive and aerospace sector. Their high specific properties makes them attractive compared to metals. Aerospace composite structures are mostly made of thermoset matrix composites processed in autoclaves. This process is costly and long for parts with complex geometries, such as variation in thickness and orientation, corners or rib features. Thus, new manufacturing technologies have to be developed to reduce the cost and processing time for complex parts. Moreover, the use of a thermoplastic matrix, instead of a commonly used thermoset matrix, can contribute to reduction of the manufacturing time, since it does not require lengthy cure cycles.

Current applications of thermoplastic composites in aerospace structures are limited to simple components with minimal curvature and thickness variations. Indeed, continuous fiber (CF) thermoplastic composites, while exhibiting good mechanical performance, are difficult to form. On the other hand, the automotive industry can produce parts with intricate features using flow molding compounds, but their fiber volume content and mechanical properties are generally too low for aerospace applications. Recent work by several authors[1-7] showed that preforms with Long Discontinuous Fibers (LDF) and high fiber volume fractions ( $>50 \%$ ) have the potential for being used in structural components due to their good forming and mechanical characteristics.

\subsection{Randomly-Oriented Strands (ROS)}

Lying between Bulk Molding Compounds (BMCs) and LDF preforms, randomly oriented strands of unidirectional prepreg composite tape (ROS), with high fiber volume fractions $(>50 \%)$, have interesting forming and mechanical characteristics [1-7]. This paper focuses on the processing of ROS thermoplastic composites by compression molding.

In this process, strands of thermoplastic composite pre-impregnated tapes are distributed randomly in a mold cavity (see Figure 1). The setup is then heated and compressed to consolidate the material. Complex shapes, including corners, thickness variations, ribs or holes, can be obtained in one compression molding step. Typically, a high pressure (60120 bar) is needed to form good quality parts $[2,6]$.

The deformation that the material undergoes during compression molding determines: (i) the feasibility of the process and (ii) the final quality of the part. Based on existing work on the deformation mechanisms encountered in randomly distributed fiber bundles forming [8-11], it is assumed that during compression molding of ROS, the material undergoes three different deformation modes:

1. An elastic compaction behavior, denoted as packing deformation, at low load. It is mostly associated to the bending and conformation of the strands. It has been studied by many authors for randomly distributed fiber bundles, Servais et al. [10] suggested an experimental method to measure the packing stress up to $100 \mathrm{kPa}$ (i.e. low normal stress).

Luchoo et al. [12] simulated the elastic response at the meso-scale. Hereunder, we consider that normal stresses $(6-12 \mathrm{MPa})$ involved while press forming ROS is way higher than the 
packing stress and that the fiber bed is fully compacted. The packing deformation therefore occurs only at very low load during the initial stage of the process and has negligible effects on the final part quality.

2. A flow at the mesoscopic scale of the strand. In this mode, the applied pressure results in a spreading of each strand. With this squeezing, the gaps between strands reduce. This mechanism is denoted as the inter-strand void content (ISVC) reduction. These interstrand voids have to reduce enough to prevent crack initiation and strength property loss in the final part. This later mechanism has been studied in a recent work [13]. An analytical model to predict the ISVC for ROS flat panels under various processing conditions and strands geometry has been developed.

3. A flow at the macroscopic scale of the part. At this scale the material can be considered as a homogeneous medium that undergoes a squeeze flow imposed by the hot press. Several analytical models and experimental procedures were developed to predict the squeeze flow of short fiber bundles for SMC or BMC molding [14, 15]. Similarly, for ROS, the viscous behavior of the equivalent homogeneous material is intimately linked to the sliding between strands. However, to the best knowledge of the authors, no extensive study of that mechanism was performed. The macroscopic squeeze flow plays a dominant role in ROS forming. It rules whether the material will flow and fill intricate features of the mold preventing short shots. These areas, such as corners or ribs, may indeed remain empty after the initial operator positioning of the strands in the mold.

The present study aims at characterizing this latter macroscopic flow phenomenon in order to better understand its sensitivity to the process parameters (temperature, pressure, strand geometry, ...). In fact, during compression molding of ROS composites, the material is deformed between two platens that are usually close to being parallel. Therefore, a study of the squeeze flow mechanism can best describe this process.

\subsection{Characterization of Composite Materials using Squeeze Flow}

In order to predict the forming of ROS composite parts, one has to study the macroscopic flow behavior at forming temperatures. Squeeze flow corresponds to a kinematic where a material is deformed between two parallel platens approaching each other at a constant applied force or closure rate [16], as shown in Figure 2. It can be used as a rheometry method for a wide range of materials, including purely viscous liquids, viscoelastic solids and purely elastic solids. Engmaan et al. [16] reviewed the major experimental and modelling techniques developed in the past years. Two configurations can be used:

- A constant material volume between the platens. The platens are larger than the squeezed sample, so the material is constrained between the platens throughout the experiment.

- A constant contact surface between the samples and the platens. The platens gap is initially fully filled with material, so the contact surface remains constant throughout the test. 
In both cases, the material is placed between the two platens and heated up to desired temperature. Once the target temperature is reached, either a constant load or a constant closure rate is applied and the load evolution or the change in height is measured over time.

For fiber reinforced thermoplastic materials, the squeeze flow test is often used to characterize the deformation occurring during processing. Shuler and Advani [17] considered fiber reinforced composites as a homogeneous continuum medium with an effective bulk transverse shearing viscosity $\eta$ [17-20]. Unidirectional fiber composite melts are assumed to behave as an incompressible anisotropic viscous fluid. Thermoplastic polymers are known to behave as a non-Newtonian, shear thinning, viscous fluid [17]. The Carreau viscosity model can be used to describe this shear thinning behavior. The flow occurs solely in the direction transverse to the fibers, since the inextensible fibers are considered to restrict flow in their direction. The flow is therefore two dimensional. Because the platen gap is usually an order of magnitude smaller than the sample width, lubrication assumption holds. Under these assumptions, the Stokes equation can easily be reduced and solved numerically to predict the sample height evolution as a function of time.

Those squeeze flow studies of UD composites under relatively low strain $(<0.5)$ can be applied to processes involving low deformation such as automated tape placement [20, 21]. In compression molding of ROS, the macroscopic flow involves large deformations ( $>50 \%$ and up to several strains). In order to develop a model for ROS, the behavior of both UD and ROS composites subjected to high pressure and large deformations are investigated in this paper, as a first step toward the development of a fully predictive model for ROS thermoplastic composites.

In section 2, the Carbon/PEEK material used, the experimental setup consisting of an instrumented hot press and the experimental procedure are presented. In section 3 , the model used to characterize the effective viscosity of UD Carbon/PEEK composites is presented. In the last section, the experimental results are discussed and show the limitation of the analytical model.

\section{Experimental Methods}

\subsection{Material}

The material used in this study is a Carbon / PEEK (PolyEtherEtherKetone) preimpregnated composite. A unidirectional (UD) $150 \mathrm{~mm}$ wide tape was used to manufacture the continuous fiber samples. ROS samples were manufactured using chopped strands of the same pre-impregnated tape. It was supplied already cut at the desired width and length. The fiber volume fraction is $60 \%$ for both materials. The consolidated ply thickness is $0.136 \mathrm{~mm}$. The manufacturer's recommended consolidation temperature is between 370 and $400^{\circ} \mathrm{C}$. Figure 3 shows $25.4 \mathrm{~mm}$ long by $6.35 \mathrm{~mm}$ wide strands. 


\subsection{Instrumented Hot Press}

A specific instrumented hot press was designed [5] (Figure 4). The fixture consists of a custom built miniaturized heated press. The testing section consists of two H13 steel platens $(100 \mathrm{~mm} \times 100 \mathrm{~mm})$ heated using four $500 \mathrm{~W}$ cartridge heaters. The platens temperature is controlled with a PID controller from Watlow. A cooling system using compressed air is also used to cool down the fixture quickly after each test. The apparatus is mounted into a $250 \mathrm{kN}$ MTS compression testing machine that allows to control the applied force. The closing of the mold is measured using the MTS transducer.

\subsection{Test Procedure}

One UD $\left[0^{\circ}\right]_{42}$ and Four ROS preconsolidated flat laminates were used to perform all the squeeze flow tests presented in section 4 . This ensured that consolidation effects at low load (packing stress) and ISVC mechanisms, discussed in section 1.1, were not observed during the experiments, but only the macroscopic squeeze flow behavior. Both the UD and ROS samples used in this study were cut from $150 \mathrm{~mm}$ by $150 \mathrm{~mm}$ flat panels preconsolidated by compression molding. The laminates were made using a steel tool with a $150 \times 150$ mm cavity.

\subsubsection{Sample preconsolidation}

For UD laminates, 42 plies of UD tape were stacked inside the cavity of the mold to reach an average consolidated thickness of $6.0 \pm 0.1 \mathrm{~mm}$. $250 \mathrm{~g}$ of chopped strands was used for each ROS laminates, to reach an average thickness of $6.5 \pm 0.2 \mathrm{~mm}$ (see Figure 1). Strands were added to the mold in small batches and shuffled manually each time to ensure random distribution and minimize their out-of-plane orientation. Then the mold was closed and placed into a Wabash 100 Tons hot press preheated to $395^{\circ} \mathrm{C}$. A thermocouple was placed into a hole on the side of the mold to measure and monitor the plate temperature during the cycle. A pressure of 22 bars was then applied and maintained during 15 minutes. The mold was then cooled down to $70^{\circ} \mathrm{C}$ at an approximate rate of $12^{\circ} \mathrm{C} / \mathrm{min}$ and was removed from the press. The panels were trimmed and $50 \mathrm{~mm} \times 50 \mathrm{~mm}$ samples were cut using a diamond blade saw.

\subsubsection{Squeeze flow tests}

The squeeze tests were all performed at $400{ }^{\circ} \mathrm{C}$ on the pre-consolidated $50 \mathrm{~mm}$ square samples. The instrumented hot-press platens were coated with FREEKOTE 700-NC release agent before each tests to prevent material sticking to the platens and ease demoulding. The samples were centered on the bottom platen and the upper platen was moved down until a contacting force was measured. The MTS machine was set in force control mode. The contacting force $(90 \mathrm{~N})$ was low compared to the applied force during the test ( 1 to $20 \mathrm{kN})$, preventing flow during the heating phase. The platens were then heated to $400^{\circ} \mathrm{C}$ in about $15 \mathrm{~min}$. Force control allows for a compensation of the thermal expansion of the setup. An additional stabilization time of 10 min ensured isothermal 
conditions during the tests. A closing force according to the test matrix given in Table 1 was then applied in a one second ramp and maintained during $5 \mathrm{~min}$. The press was cooled down using compressed air. The cooling rate was not controlled but measured to be approximately $15^{\circ} \mathrm{C} / \mathrm{min}$. Finally, when the temperature dropped below $143^{\circ} \mathrm{C}$, which corresponds to the glass transition temperature of PEEK, the press was opened and the sample ejected. Sample length, width and thickness were measured prior and after each squeeze flow test, using a micrometer and caliper.

Four different strands size were tested, and four different load levels were used for each strand size (see Table 1). The load levels are such that the material remained inside the press platens, ensuring a constant volume experiment, during the whole squeeze flow test.

\subsection{Data acquisition}

Force and relative displacement signals were acquired, at a rate of $10 \mathrm{~Hz}$, during the whole test. The MTS machine transducer provided the relative sample height evolution. The actual sample height evolution $h(t)$ was obtained by compensating for the material and fixture thermal expansion. The final sample thickness $h_{f}$ was measured at room temperature using a micrometer. Because of the thermal expansion between room and processing temperature, corrections had to be performed. $\varepsilon_{t h}$ is the transverse coefficient of thermal expansion of the material integrated between room temperature and processing temperature $\left(400^{\circ} \mathrm{C}\right)$. The final height of the specimen at processing temperature $h_{f}^{400}$ writes:

$$
h_{f}^{400}=h_{f}\left(1+\varepsilon_{t h}\right)
$$

$\varepsilon_{t h}$ was measured at $8 \%$ using a TA instruments Q400 thermomechanical analyzer on a quasi-isotropic laminate, and is supposed to be similar for UD and ROS specimens (transverse behavior).

The sample final height at processing temperature $h_{f}^{400}$ was used to offset the acquired relative displacement measurement and obtain absolute height change data. The final height was measured for every sample and compared to the initial height $h_{i}$ to obtain the final strain:

$$
\varepsilon_{f}=1-h_{f} / h_{i}
$$

\section{Modelling}

Considering a two dimensional transverse flow, the model of Shuler and Advani [17] is adapted for a constant material volume configuration (see Figure 2). 


\subsection{Squeeze flow under lubrication assumption}

Under lubrication assumption the vertical component of the velocity is neglected versus its horizontal $x$ coordinate $V_{x}(x, y)$. The velocity derivative versus the $x$ dimension can also be neglected compared to its $y$ derivative. The pressure field $P$ can be considered constant through thickness in the $y$ direction. The Stokes equation then reduces to [17]:

$$
\frac{\partial P(x)}{\partial x}=\eta \frac{\partial^{2} V_{x}(x, y)}{\partial y^{2}}
$$

where $\eta$ is viscosity, that can be modeled using the Carreau law:

$$
\eta=\eta_{0}\left[1+(\lambda \dot{\gamma})^{2}\right]^{(n-1) / 2}
$$

where $\eta_{0}$ is the zero shear rate viscosity, $n$ is the shear thinning exponent and $\lambda$ is the relaxation time.

As for the boundary conditions, according to previous experimental work [5], no slip conditions are imposed at the platen interface:

$$
V_{x}(x, y)=0 \quad \text { at } y=h(t) / 2
$$

Using symmetries, only one quarter of the geometry is modelled, and at $\mathrm{y}=0$ :

$$
\frac{d V_{x}(x, y)}{d y}=0
$$

The closure force $F(t)$ equates the pressure $P(x)$ integral over the platen surface:

$$
F(t)=\int_{0}^{2 L} \int_{0}^{W} P(x) d x d z=2 W \int_{0}^{L} P(x) d x
$$

The effective pressure $P_{\text {eff }}$ is also defined based on the sample area evolution:

$$
P_{\text {eff }}=\frac{F(t)}{L(t) W}
$$

Finally, the sample volume is assumed constant due to material incompressibility, such that:

$$
L(t)=\frac{h_{0} L_{0}}{2 h(t)}
$$


where $L_{0}$ and $h_{0}$ are respectively the initial sample length and height. Considering a control volume between the vertical planes at coordinate 0 and $x$, incompressibility also gives:

$$
\int_{0}^{x} \frac{d h(t)}{d t} d x=\frac{d h(t)}{d t} x=\int_{0}^{h / 2} V_{x}(x, y) d x
$$

\subsection{Numerical Implementation}

The time coordinate was discretized using a constant time step $d t$. Space is discretized using a regular grid with horizontal and vertical spacing $d x$ and $d y$ as shown in Figure 2. At each time step, the pressure field versus $x$, the horizontal velocity field $V_{x}(x, y)$ and the closure rate $d h(t)) / d t$ are solved for. To this end, an unknown vector $\{X\}$ is defined as the concatenation of the horizontal velocities $V_{x}(x, y)$ at each node, the pressure $P(x)$ at each $x$ node and the platens closure rate $d h(t)) / d t$ :

$$
\{X\}=\left\{\begin{array}{c}
V_{x}\left(x_{1}, y_{1}\right) \\
V_{x}\left(x_{1}, y_{2}\right) \\
\vdots \\
V_{x}\left(x_{1}, y_{n}\right) \\
P\left(x_{1}\right) \\
V_{x}\left(x_{2}, y_{1}\right) \\
V_{x}\left(x_{2}, y_{2}\right) \\
\vdots \\
V_{x}\left(x_{2}, y_{n}\right) \\
P\left(x_{2}\right) \\
\vdots \\
P\left(x_{m}\right) \\
\frac{d h(t)}{d t}
\end{array}\right\}
$$

Finite differences are used to discretize equation (3), (4), (5) and (6) and numerical summation are used to implement equations (7) and (10).

The system of equations (3) to (11) can then be written in the residual form:

$$
\left\{R\left(X, F, h_{0}, L_{0}, \eta_{0}, \lambda, n\right)\right\}=\{0\}
$$

These $m \times(n+1)+1$ equations are solved using a Levenberg-Marquardt nonlinear solver. It is called within MATLAB with the $f$ solve command. This non-linear problem appears within the time integration scheme and gives the solution at next time step, given a state at current time step. Thus, using the current solution as an initial guess, the solution will only slightly differ. The nonlinear solver method appears very robust in this case. At each time step, $d h(t) / d t$ is thus obtained, and the thickness evolution can be computed with an explicit Euler integration scheme and the initial thickness $h_{0}$. Note that the geometry is updated at each time step using equation (9). 


\subsection{Parameter identification}

The evolution of the sample height $h(t)$ was compared to the experimental data obtained from the instrumented hot press. An inverse method, using a least square fit, gives the optimal three Carreau parameters $\left(\eta_{0}, \lambda, n\right)$. The optimum was obtained using the builtin simplex search method in MATLAB.

The optimal Carreau parameters were found once the average error between the experimental data and the model was below $5 \%$ for all the load levels. In order to obtain an optimal solution, only the first 5 seconds of each test were considered, where the viscous phase was dominant. In fact, the MATLAB simplex search method could not converge for the complete test duration (300 seconds), since the Carreau model does not capture the plateau behavior that was observed experimentally. This latter aspect will be further discussed in section 4.1.

Also, the obtained parameters were compared to the literature for similar materials and test conditions [17], and the order of magnitude was in good agreement. This suggests that the obtained parameters are significant and are physically meaningful. . Table 2 shows the numerical model input parameters used, and the obtained Carreau parameters.

\section{Results and Discussion}

In this section, the experimental and modelling results obtained following the procedures presented in section 2 and 3 are presented and discussed.

\subsection{Global flow description and model comparison with experimental data}

Figure 5 shows sample height changes as a function of time for the 4 different load cases, for ROS samples with small 6.35 by $3.18 \mathrm{~mm}$ strands. It gives an overview of the global flow behavior under high pressure. Initially, the material is fully melted under isothermal conditions. The contact load is not high enough to create flow. At time $t=0 \mathrm{~s}$, when the force is applied in a one second ramp, the material starts to flow. The effective pressure resulting from the closure force (equation (8)) is then maximum. This is shown in Figure 6 where the effective pressure $\left(P_{e f f}\right)$ evolution is plotted as a function of time for the different load levels on UD samples. Then, the sample area increases as the material flows, and the effective pressure reduces. It eventually reaches a minimum pressure $P_{\min }$ not sufficient to reach the yield stress and further deform the material: the sample thickness reaches a plateau. The final time $t_{\min }$ is defined as the time needed to reach this plateau.

The slopes of sample height as a function of time show that the flow velocity strongly depends on the applied load. For the load range tested ( 1 to $20 \mathrm{kN}$ ), the yield stress was always reached before two minutes.

Figure 7 shows a comparison between the modelled and the experimental sample height versus time for different closure forces on UD samples. The model fails to predict accurately the complete squeeze flow under those load levels. The strain is high for the model, compared to typical strains of a few $\%$ in the literature [20]. However, Figure 8 shows that during the first seconds, the model reasonably reproduces the sample height evolution over time. 
The critical viscous time $t_{c}$ is defined as the time when the predicted height starts to deviate by more than $5 \%$ from the measured one. $t_{c}$ is an indicator of the duration of the fluid behavior. It determines until when the assumption of an equivalent fluid behavior for the composite melt is valid. It also corresponds to the time range where the Carreau model can be accurately used to predict the sample height reduction. Also, the viscous strain fraction $\alpha_{\mu}$ is defined as the ratio between the predicted height after $t_{c}\left(h\left(t_{c}\right)\right)$ and the final height $h_{f}$, with respect to the initial height $h_{0}$ :

$$
\alpha_{\mu}=\left(\frac{h_{0}-h\left(t_{c}\right)}{h_{0}-h_{f}}\right)
$$

Figure 9 shows $t_{c}$ and $\alpha_{\mu}$ as a function of the applied force for the UD material. $t_{c}$ is less than 5 seconds for the load range tested in this study. $\alpha_{\mu}$ quantifies how much of the total deformation occurs during the fluid phase. In the case presented above, $\alpha_{\mu}$ ranges between $35 \%$ to $80 \%$. On the one hand it shows that assuming an equivalent bulk viscosity for the composite melt is valid during only a few seconds of the process, but on the other hand most of the deformation occurs during that time for high loads.

\subsection{Comparison between ROS and UD}

For UD $\left(0^{\circ}\right)$ preconsolidated laminate, the flow only occurred transversely to the fiber direction, while for ROS samples, flow occurred in both $x$ and $z$ direction (see Figure 10). The 1-D symmetric transverse flow observed for UD samples validates the assumption used in the model. However, a 2-D flow was observed for ROS samples, which makes the 1-D assumption invalid in that case. Moreover, since the model can only predict the first seconds of a test, as shown above, it was not applied to squeeze flow of ROS.

Figure 11 shows the sample height evolution as a function of time for different strands size as well as UD samples, using a closure force of $5 \mathrm{kN}$. For large strands $(25.4 \times 6.35 \mathrm{~mm})$ the flow is much slower at that load level and the final thickness is twice higher than for smaller strands. Also, UD and small strands sample thickness evolutions match during the first seconds. Then, at $t_{\text {min }}$, when the pressure reaches the minimum pressure $P_{\text {min }}$, and the yield stress is reached, the flow stops, and the sample height remains constant. $t_{\min }$ is generally increasing with strands size, and decreasing with the applied load. However, due to the heterogeneity of ROS, there is some discrepancy in the results, as shown on Figure 11 , where the final height reached for small strands $(6.35 \times 3.18 \mathrm{~mm})$ is a bit higher than medium strands.

\subsection{Strands size effect}

Figure 12 shows the load versus final strain (equation 2) curves for the different load level tested in this study. The larger the strands, the higher the pressure required to reach a given strain. It shows that strand size has a large effect on the material behavior.

Except for one outlier at $5000 \mathrm{~N}$ for the 12.7 by $3.18 \mathrm{~mm}$ strands, the behavior of the two medium size strands is similar. Nonetheless, there is a consistent final strain increase with the strand length decreasing from 25.4 to 12.7 to $6.35 \mathrm{~mm}$. This suggests that the final 
strain depends mostly on strand length, and less on strand width. This phenomenon was expected, since it is known that polymer composites viscosity and yield stress are greatly affected by the fibres dimensions and architecture. Therefore, short strands samples have lower viscosity and yield stress and results in higher strain.

At low closure force, the final strain is more sensitive to strands length $(0.5$ strain difference at $1000 \mathrm{~N}$ ), whereas at higher load the final strains are closer (within 0.2 strain). Moreover, the non-linear behavior observed on Figure 12 suggests that a maximum final strain of around 0.8 cannot be exceeded, even with higher forces. This is the upper limit for the macroscopic squeeze flow of the $50 \times 50 \times 6.5 \mathrm{~mm}$ flat panel at $400^{\circ} \mathrm{C}$, investigated in this study.

\subsection{Repeatability}

The experimental results presented for the medium slender strand size (12.7 $\mathrm{mm} \times 3.18$ $\mathrm{mm})$ and large strand size $(25.4 \mathrm{~mm} \times 6.35 \mathrm{~mm})$ were repeated three times per test conditions, and the average and standard deviation values were calculated for these two strand sizes. Figure 12 shows the average values obtained for the three repeated test conditions for the large and medium slender strands, and also incorporates the standard deviation error bars.

For the medium slender strands the standard deviation is approximately $2 \%$ for the three higher load levels and 5\% for lower load levels. This suggests that the dimension of the samples is large enough to overcome the material variability. For large strands, larger variability was found in the results where an average standard deviation of 5\% was observed with values up to $7 \%$. These results were expected, as larger strands are more prone to heterogeneity in a sample having the same dimensions.

\section{Conclusion}

An experimental characterization of the squeeze flow mechanism was performed using an in-house developed instrumented hot press. Also, the analytical model available in the literature was used. The previous models were mainly developed for composite manufacturing processes that involve lower strains, such as Automated Tape Placement. Those models were relatively accurate to predict the squeeze flow behavior of unidirectional thermoplastic composites under moderate strain $(<50 \%)$. But it fails to predict the large strain phase behavior ending with a plateau.

High pressure flow of UD or ROS composite materials is mainly governed by 2 regimes.

- A Non-Newtonian fluid behavior of the composite melt with an equivalent viscosity. It is dominant in the first seconds of the process, when the pressure is maximum and the strain rate is low. The effective pressure progressively decreases during this phase. 
- A second yielded phase. When the composite melt has reached a given strain, the shear rate quickly drops to nearly zero and the flow stops. This confirms the existence of a yield stress, likely linked to the inter-ply friction behavior.

Quantitative indicators were defined to analyze those two phases. The viscous strain fraction $\boldsymbol{\alpha}_{\boldsymbol{\mu}}$ showed that most of the deformation is fluid like. The final time $\boldsymbol{t}_{\min }$ indicated that the squeeze flow is fast and occurs in less than 2 minutes even for the largest strands.

The strand size, and especially the strand length, have a large effect on the squeeze flow mechanism.

This study showed that forming UD and ROS Carbon/PEEK composites under high pressure involves two phenomena. To predict the forming of ROS complex parts under high pressure, new models taking both the viscous and the inter-ply friction behavior have to be developed. In order to accurately predict the press forming of ROS, the material behavior needs to incorporate those yield effects, governing the minimum pressure at which the material starts to flow.

\section{Acknowledgements}

The authors would like to greatly acknowledge financial support provided by McGill University and the industrial partners: Bell Helicopter Textron Canada Limited, Bombardier Inc., Pratt and Whitney Canada Corp., Hutchinson Aerospace-Industry, Delastek Inc. and Avior Integrated Products Inc. The authors would also like to acknowledge Steven Roy from National Research Council Canada, as well as Quentin Fabien, Benoit Landry and Dominic Leblanc from McGill University for their help in the design, manufacturing and calibration of the instrumented hot press. Benoit Landry also performed the thermal expansion measurements. The authors would also like to acknowledge the funding provided by the Natural Sciences and Engineering Research Council (NSERC) and the Consortium for Research and Innovation in Aerospace in Quebec (CRIAQ). 


\section{References}

1. P. Feraboli., et al., Journal of Reinforced Plastics and Composites, 28, 10 (2008).

2. M. van Wijngaarden, A. Jongbloed, and J. de Vries, Proceedings of the SAMPE Conference. Seattle, USA (2010).

3. M. Selezneva, et al., Proceedings of the SAMPE Conference. Baltimore, USA, (2012).

4. G.-P. Picher-Martel, and P. Hubert, Proceedings of the American Society for Composites Conference. Arlington, TX, USA, (2012).

5. G.-P. Picher-Martel, A. Levy, and P. Hubert, Proceedings of the 19th International Conference on Composite Materials, Montréal, Canada, (2013).

6. N. Eguèmann, et al., Proceedings of the 19th International Conference on Composite Materials. Montréal, Canada, (2013).

7. D. LeBlanc, et al., Proceedings of the SAMPE Technical Conference, Seattle, USA, (2014).

8. C. Servais, A. Luciani, and J.-A.E. Månson, Journal of Rheology, 43, 4 (1999).

9. C. Servais, A. Luciani, and J.-A.E. Månson, Journal of Non-Newtonian Fluid Mechanics, 104, 2-3, (2002).

10. C. Servais, V. Michaud, and J.-A.E. Månson, Polymer Composites, 22, 2 (2001).

11. P. Dumont, et al., Composites Part A : Applied Science and Manufacturing, 38, (2007).

12. R. Luchoo et al., Proceedings of the 18th International Conference on Composite Materials. Jeju Island, South Korea (2011).

13. A. Levy, and P. Hubert, Composites Part A: Applied Science and Manufacturing, (under review).

14. M. Sepehr, G. Ausias, and P.J. Carreau, Journal of Non-Newtonian Fluid Mechanics, 123, 1, (2004).

15. M. Sepehr, et al., Journal of Rheology, 48, 5, (2004).

16. J. Engmann, C. Servais, and A.S. Burbidge, Journal of Non-Newtonian Fluid Mechanics, 132, 1-3, (2005).

17. S.F. Shuler, and S.G. Advani, Journal of Non-Newtonian Fluid Mechanics, 65, 1, (1996).

18. J.A. Goshawk, V.P. Navez, and R.S. Jones, Journal of Non-Newtonian Fluid Mechanics, 73, 3 (1997).

19. H.M. Laun, M. Rady, and O. Hassager, Journal of Non-Newtonian Fluid Mechanics, 81, 1-2, (1999).

20. E.L.Wang, and T.G. Gutowski, Composites Manufacturing, 2, 2, (1991).

21. S. Ranganathan, S.G. Advani, and M.A. Lamontia, Journal of Composite Materials, 29, 8, (1995). 
Picher-Martel, Fig. 1: Randomly oriented strands ready to be processed in a flat panel.

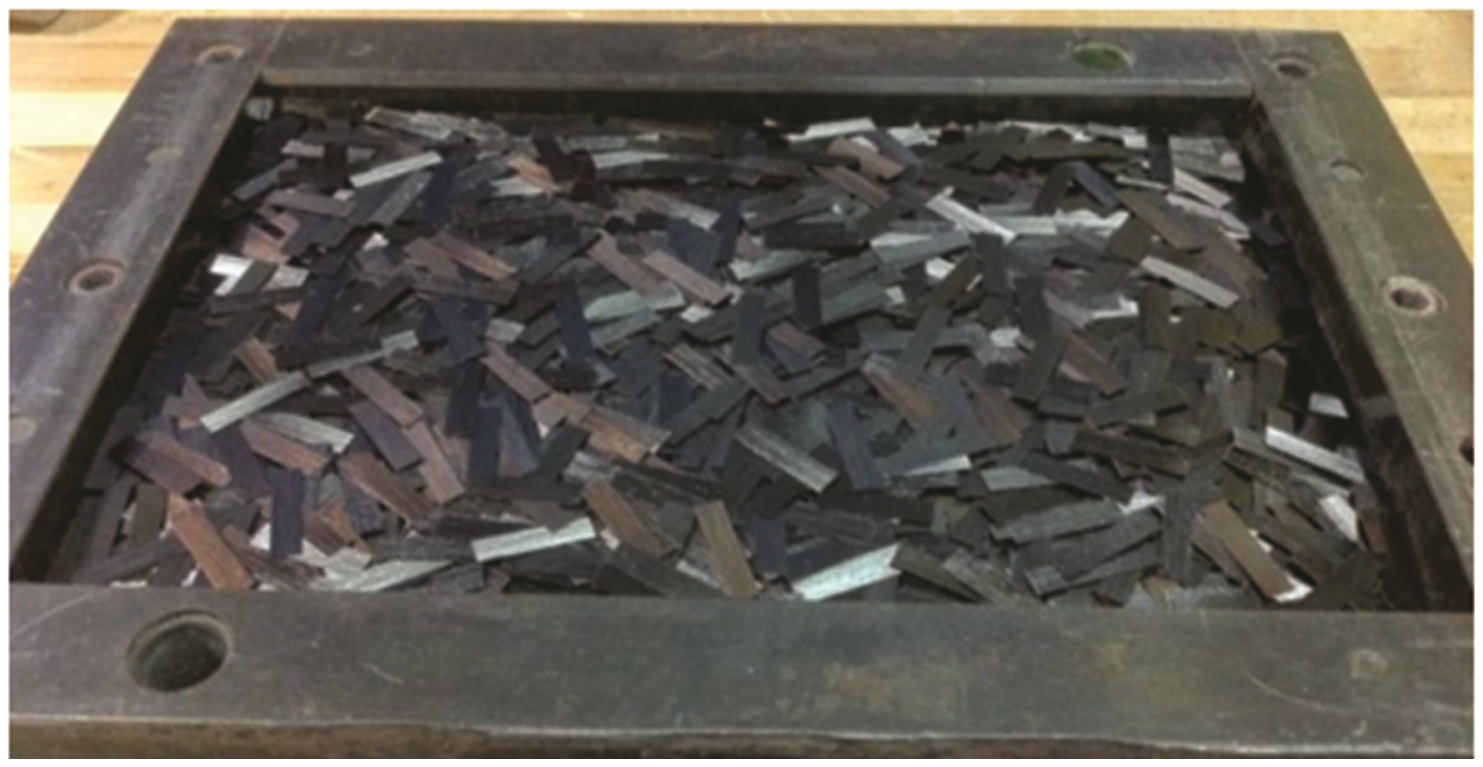




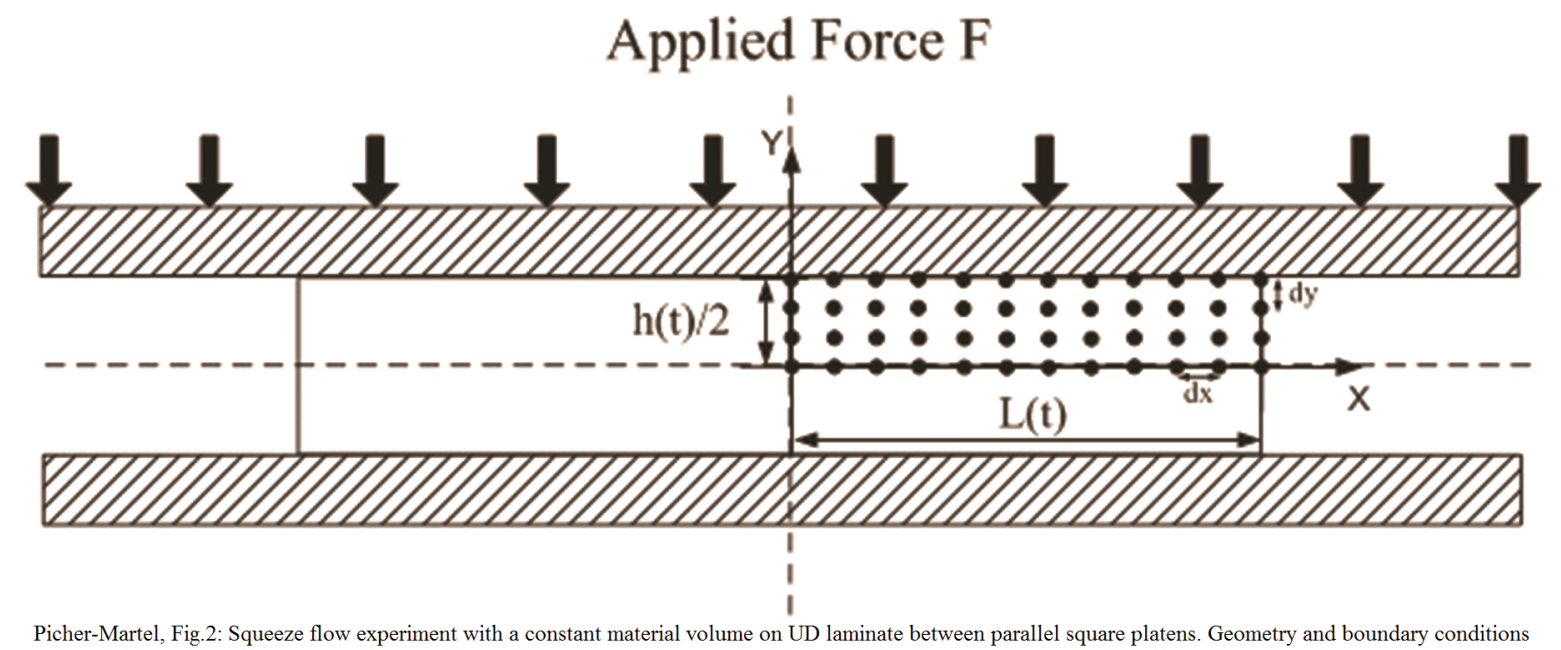
considered in this study. 
Picher-Martel, Fig.3: 25mm long strands used to make the ROS panels.

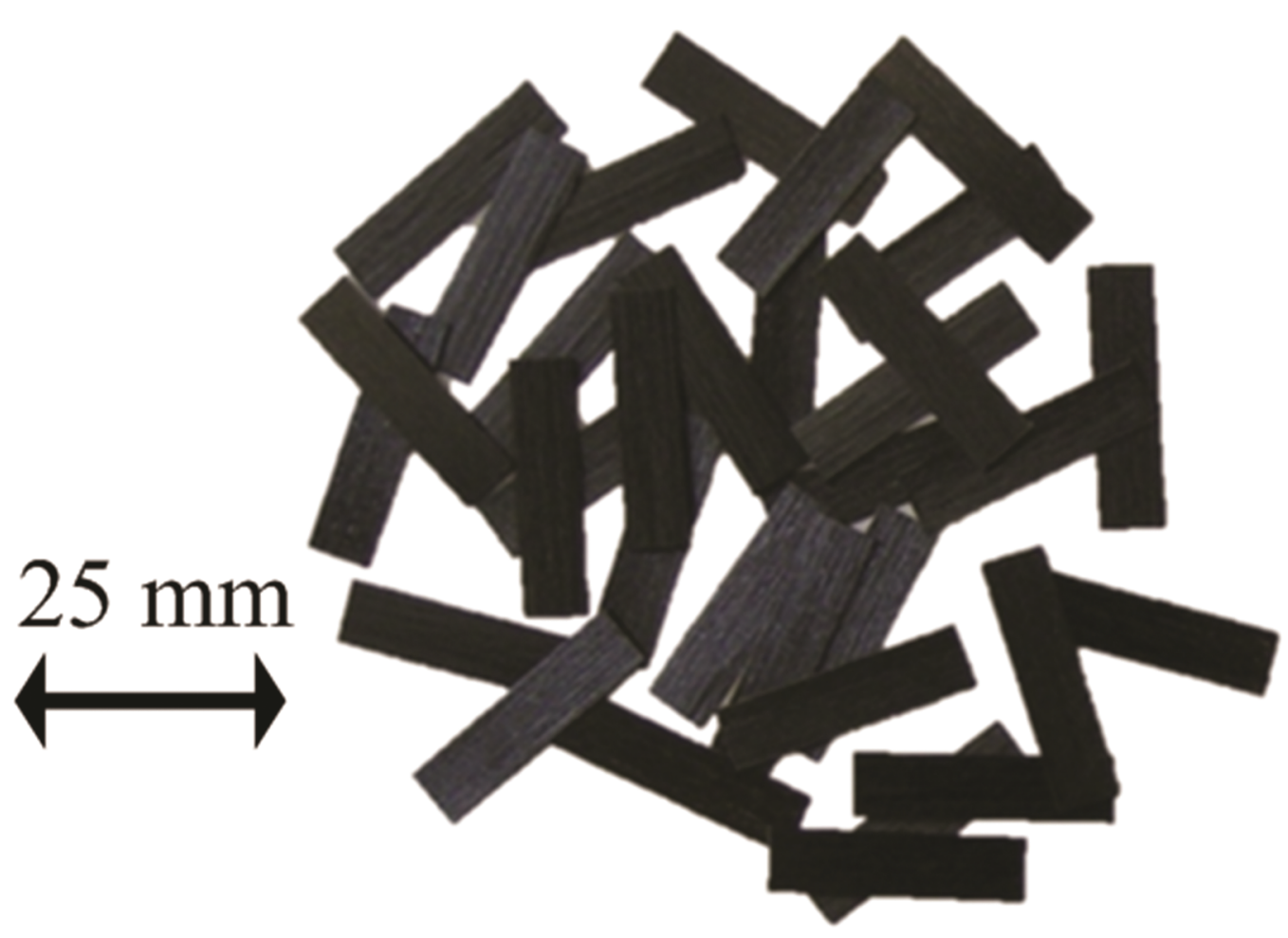


Picher-Martel, Fig.4: Instrumented hot press. (1) MTS compressive fixture. (2) Ball-bearing die set to ensure alignment. (3) H13 steel $100 \mathrm{~mm}$ x $100 \mathrm{~mm}$ platens (4) $500 \mathrm{~W}$ Heating cartridges. (5) Cooling channels using compressed air. (6) Ceramic layer to ensure thermal insulation. (7) A picture frame can be used to pre-consolidate flat panels. (8) Material placed inside the mold

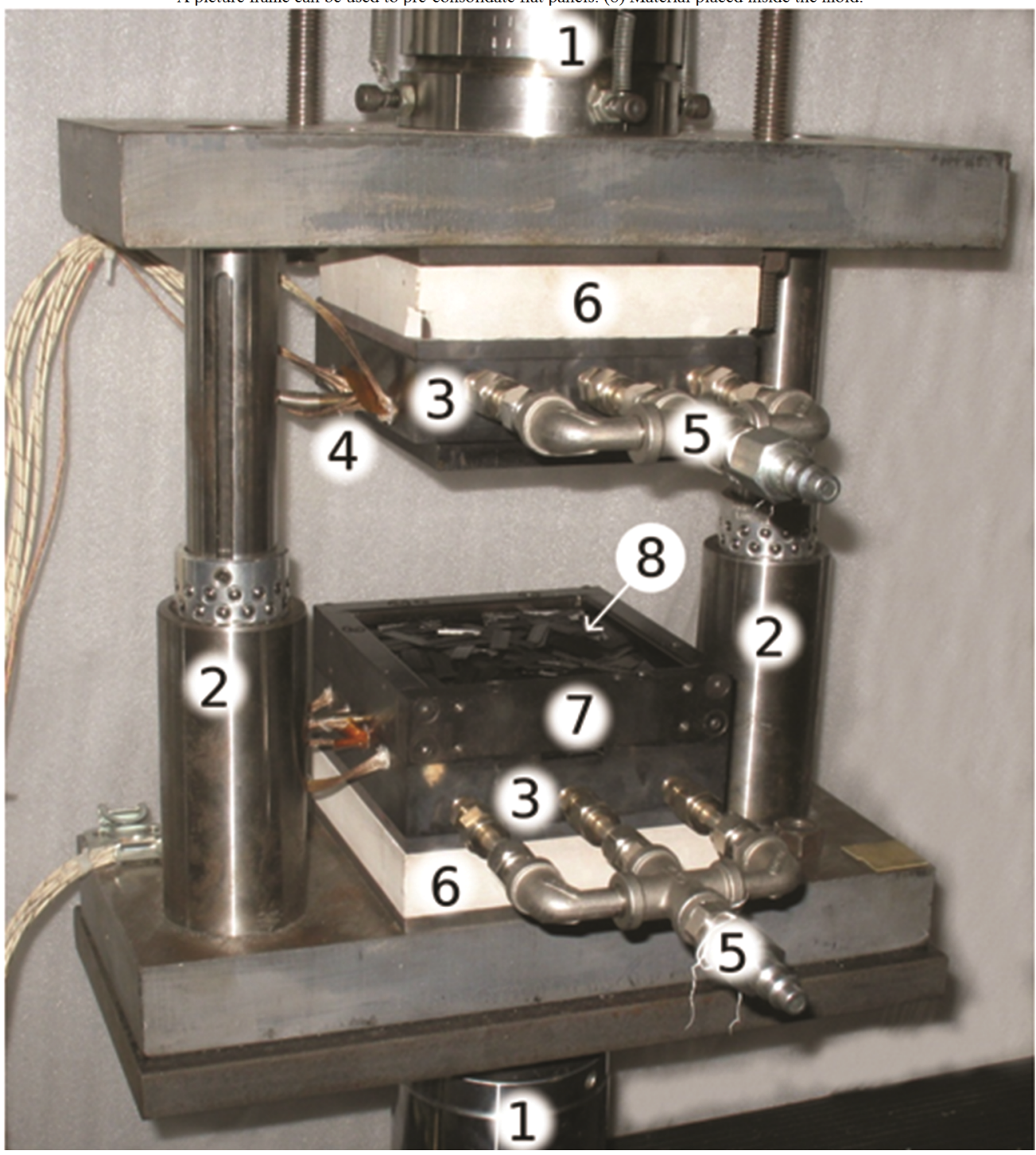




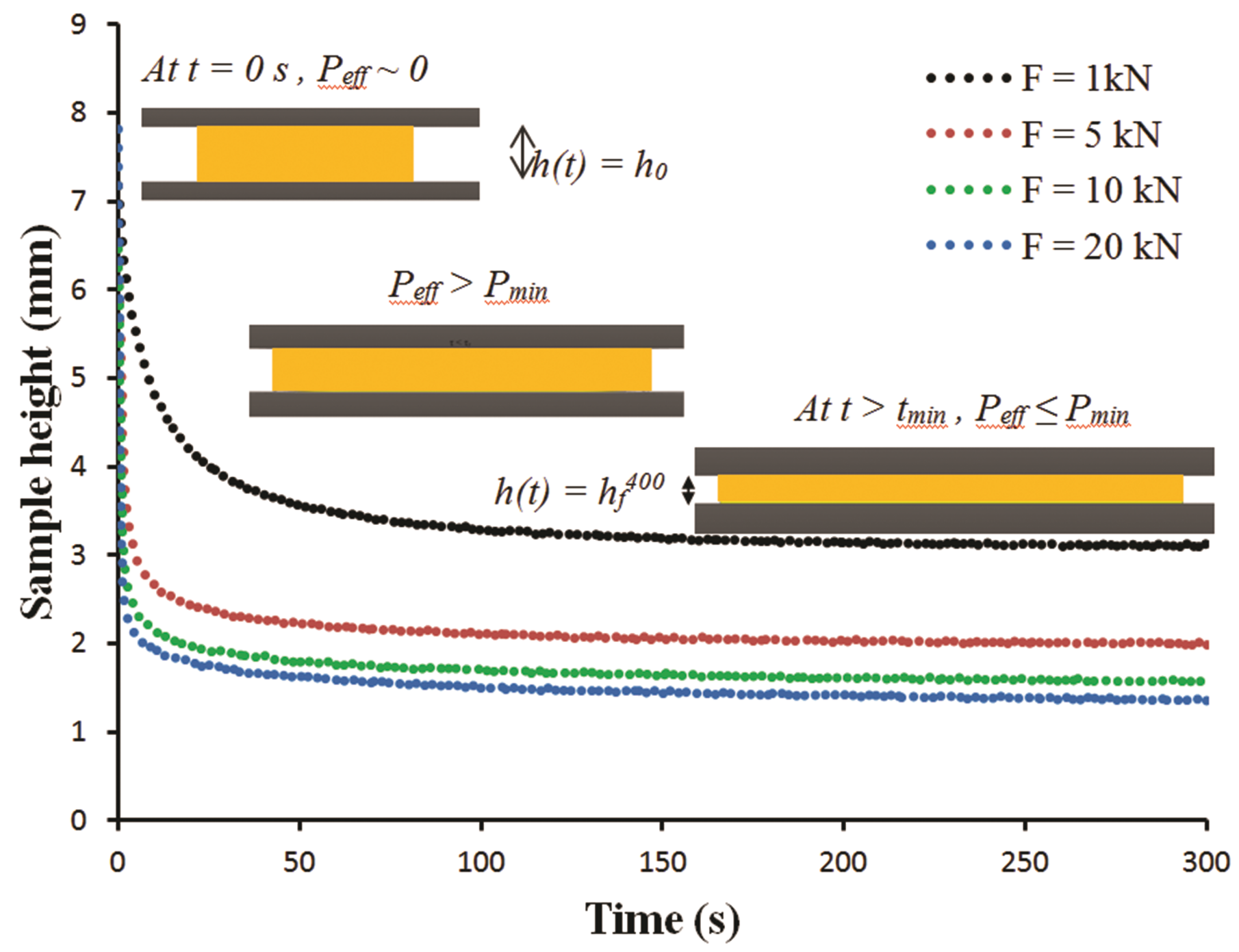

Picher-Martel, Fig.5: Sample thickness reduction as a function of time, for different closure forces, for ROS samples. Strands size : $6.35 \times 3.18 \mathrm{~mm}$. Two phases are visible: viscous fast squeeze an unyielded plateau. 


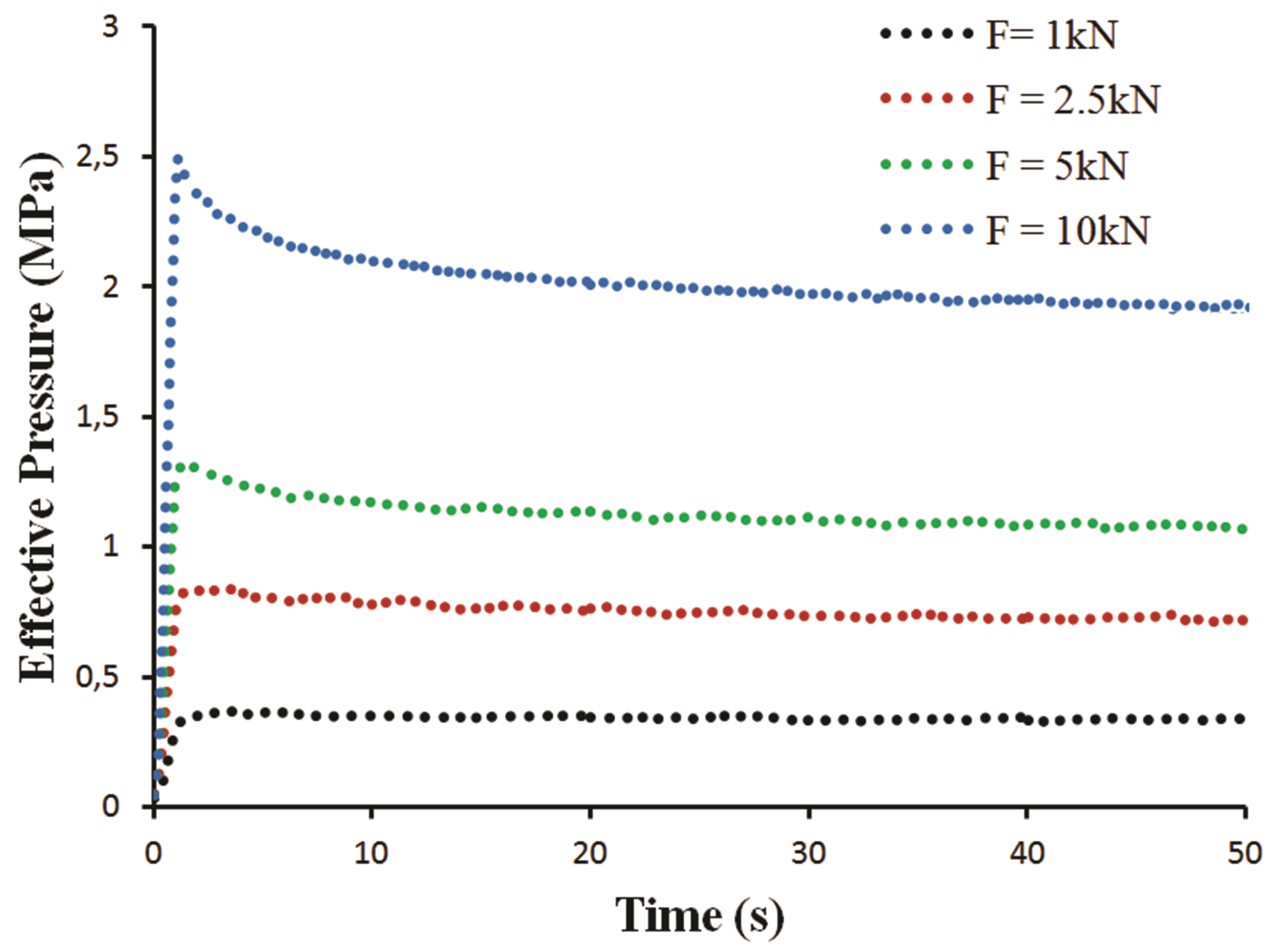

Picher-Martel, Fig.6: Effective pressure evolution as a function of time for different closure forces on UD samples. After the initial increase associated with the force imposition, the effective pressure decreases as the sample area increase. 


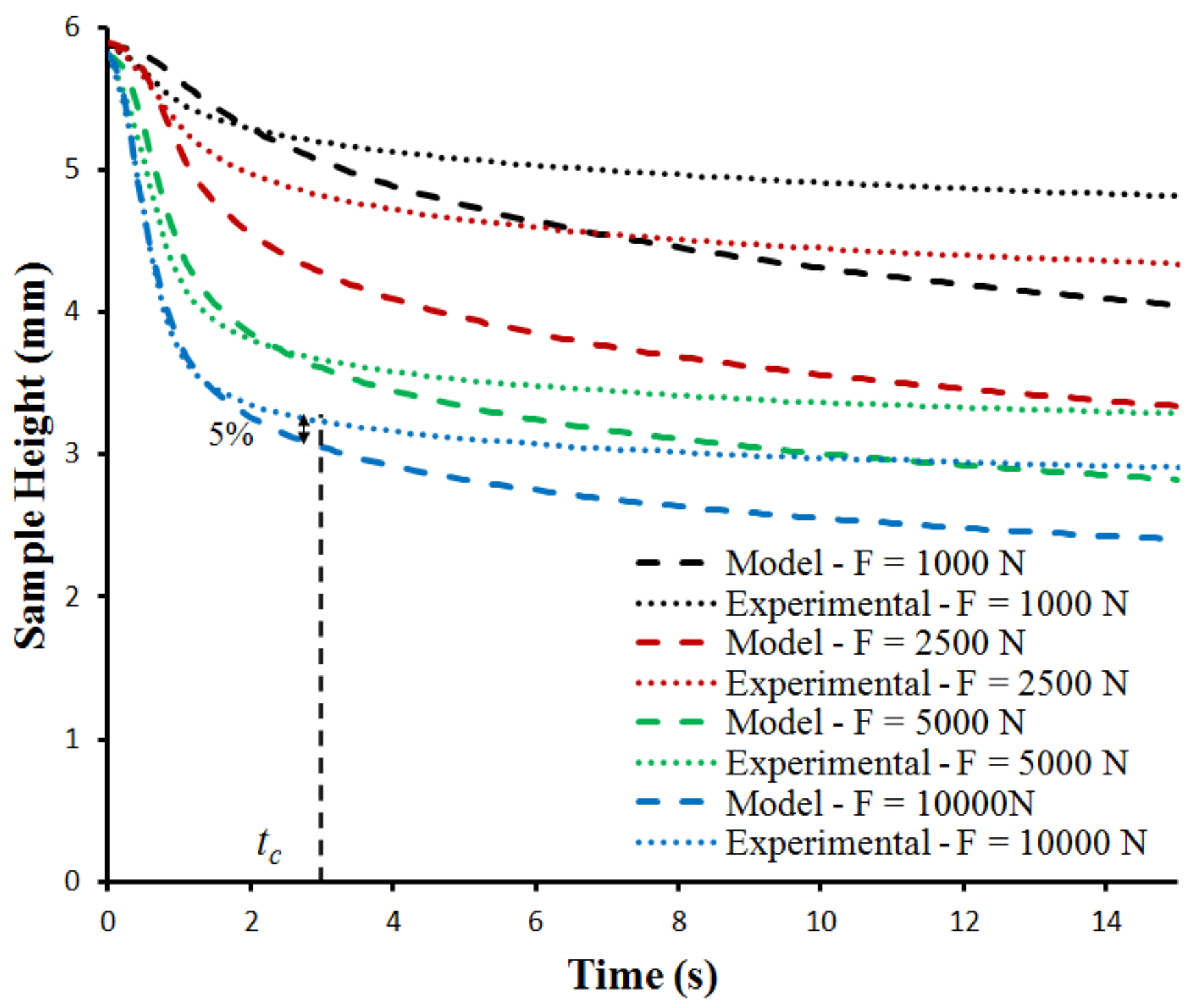

Picher-Martel, Fig.7: Comparison between analytical model and experimental results on UD samples during a complete squeeze flow test. Discrepancy between the model and the experimental data appears after a few seconds. $t_{c}$ is shown for the $10000 \mathrm{~N}$ case. 


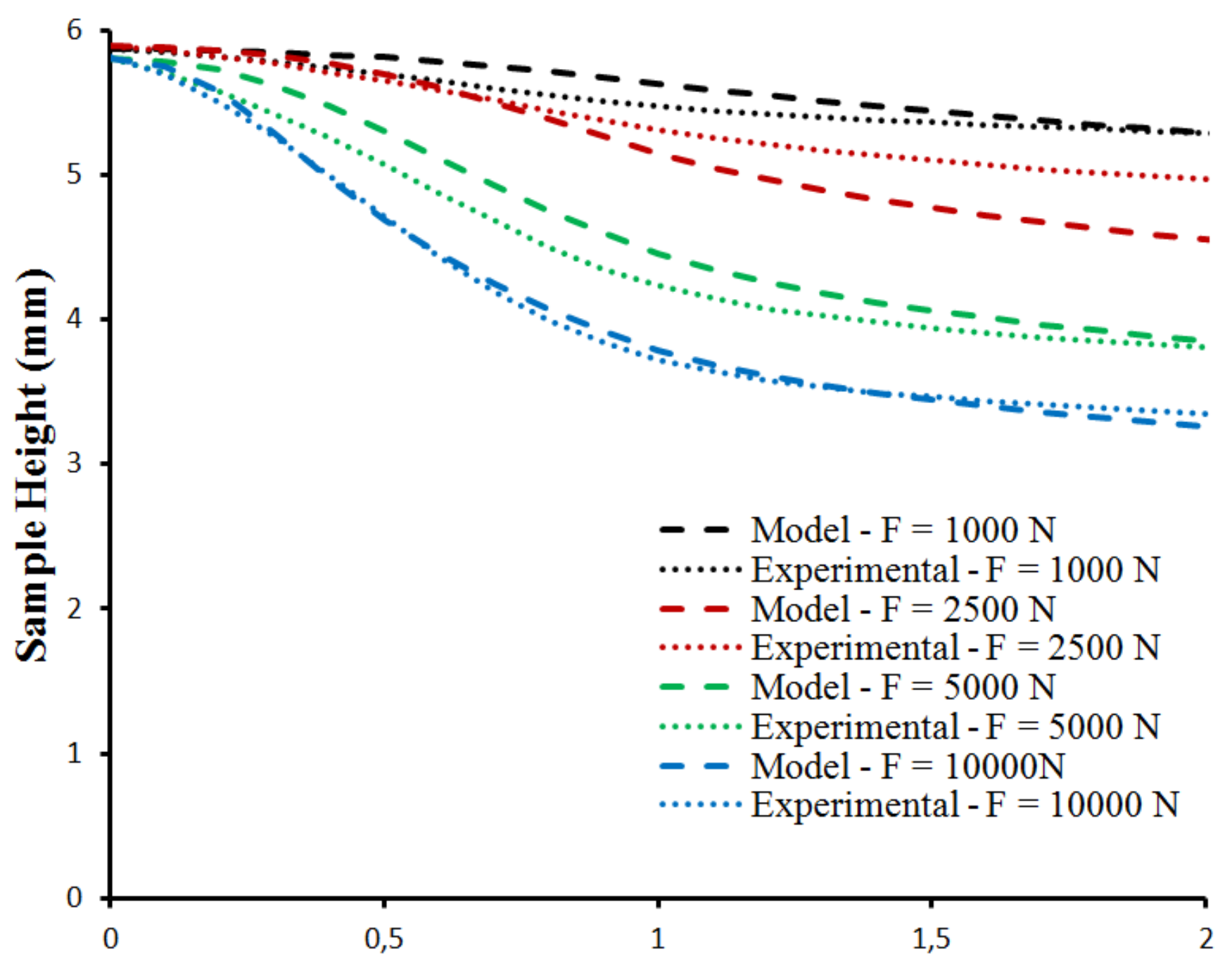

\section{Time (s)}

Figure 8: Comparison between analytical model and experimental results on UD samples during the first seconds of a squeeze flow test. The model is more accurate during this viscous phase. 


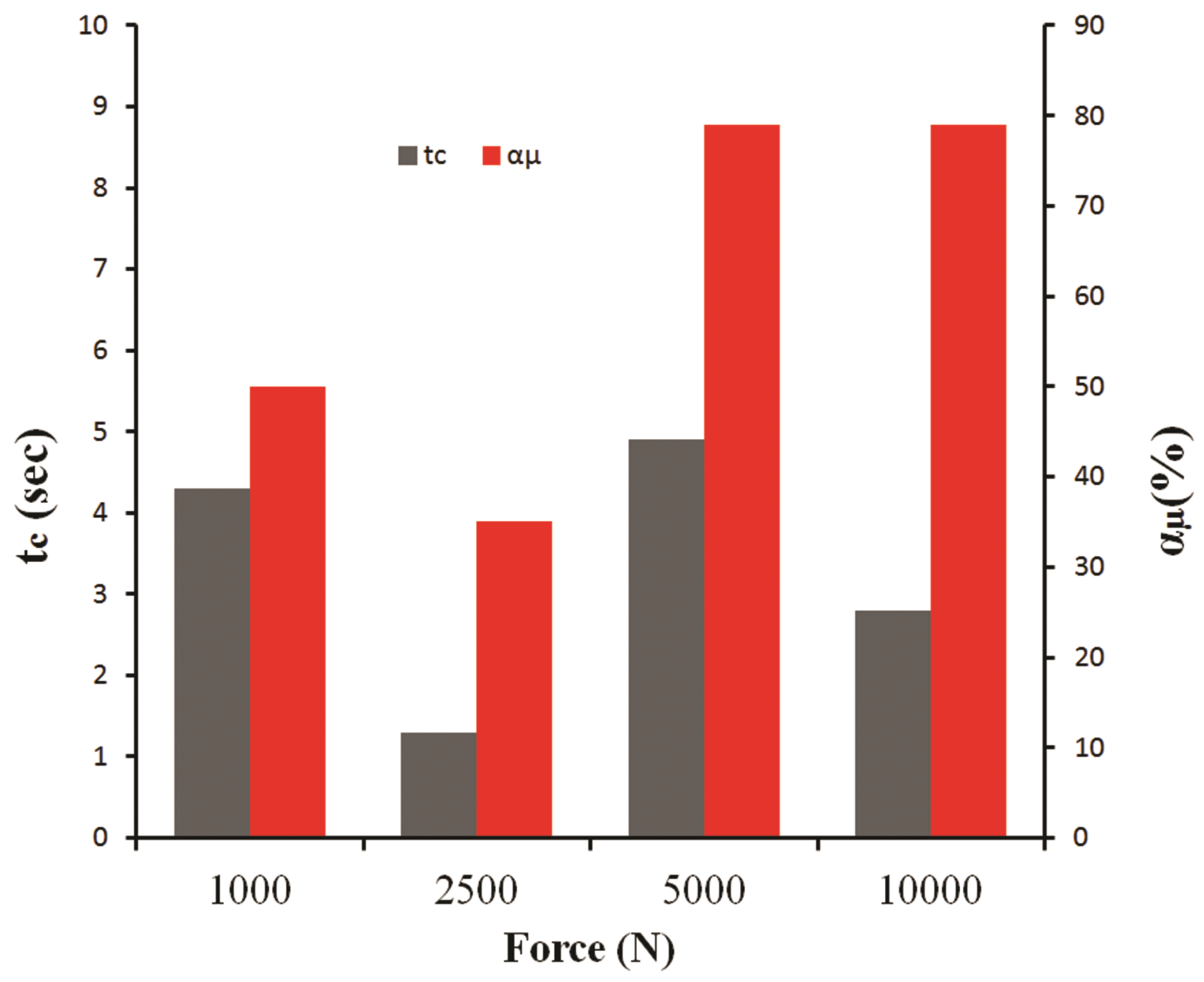

Picher-Martel, Fig.9: Critical viscous time and viscous strain fraction for the squeeze flow of unidirectional samples for the four closure forces. Most of the deformation occurs during the fast viscous phase. 
Picher-Martel, Fig. 10: Pictures of a UD sample a) prior to squeeze flow b) after squeeze flow and a ROS sample c) prior to squeeze flow d) after squeeze flow. UD exhibits a 1D transverse flow, ROS a bidirectional flow.

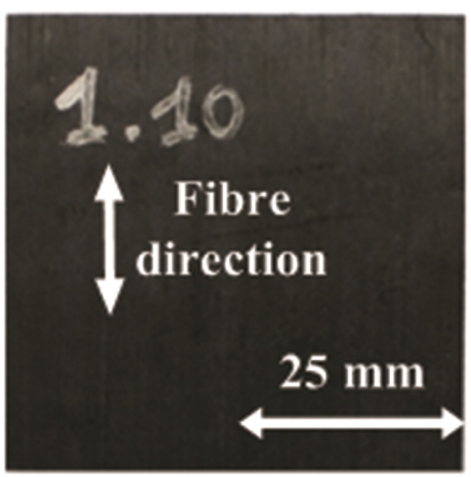

a)

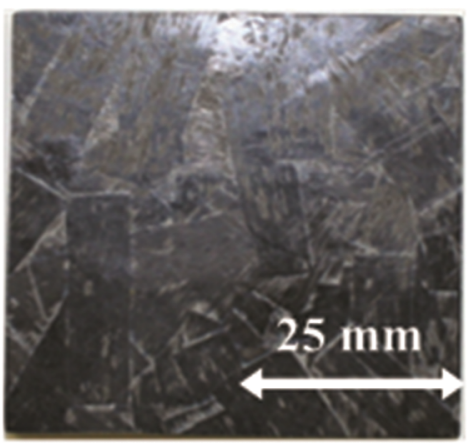

c)

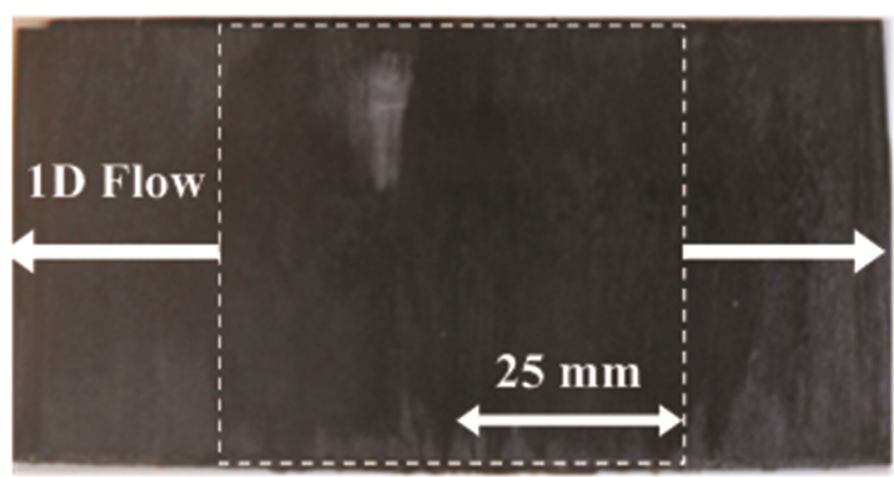

b)

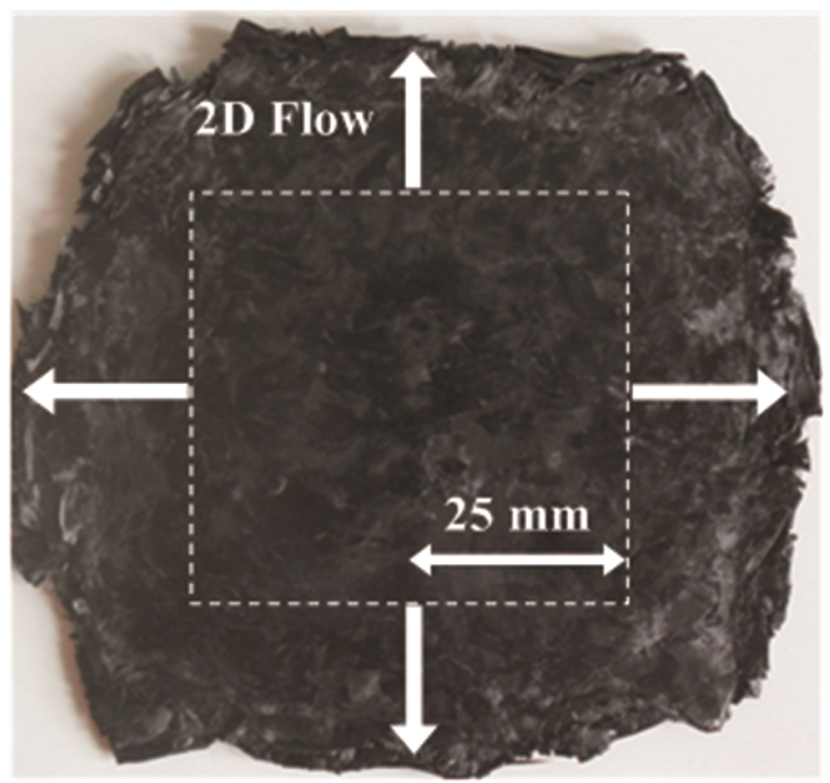

d) 


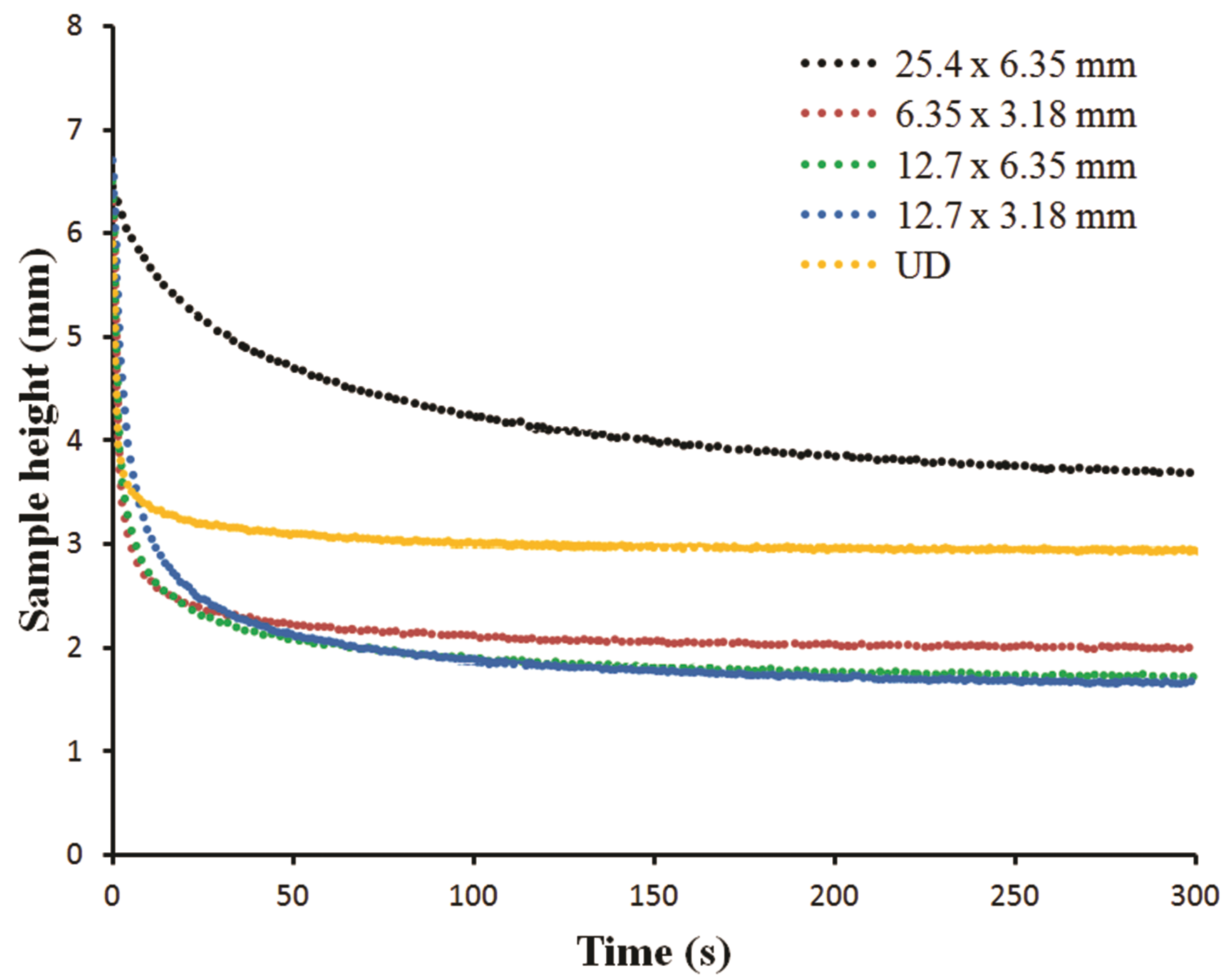

Picher-Martel, Fig. 11: Comparison between different ROS and UD samples for a closure force of $5 \mathrm{kN}$. 


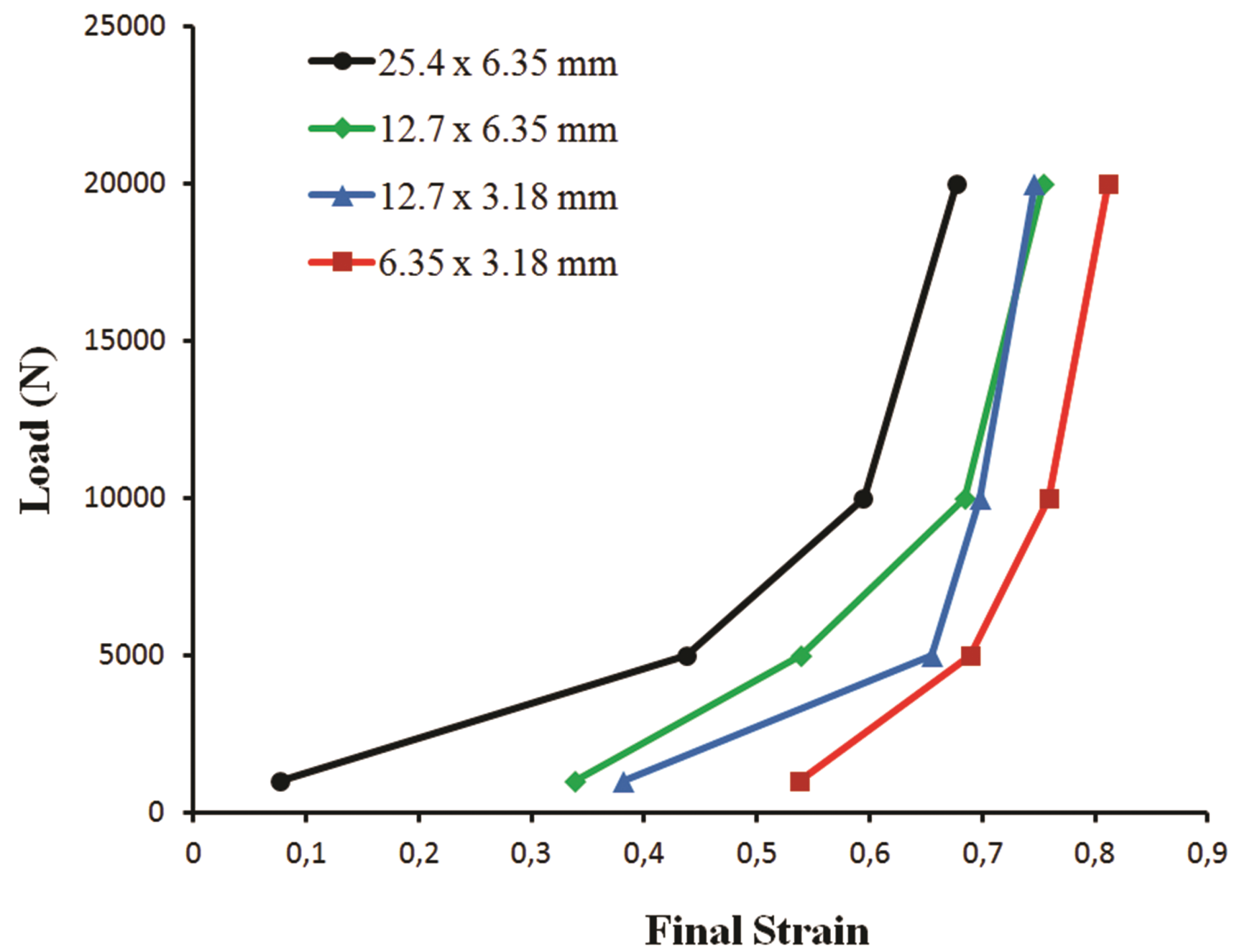

Picher-Martel, Fig.12: Load as a function of final strain for different strand size. Final strain depends on strand length and $80 \%$ strain cannot be exceeded. 
Table 1 - Experimental test matrix

\begin{tabular}{ccccc}
\hline Material & $\begin{array}{c}\text { Strand } \\
\text { length } \\
(\mathrm{mm})\end{array}$ & $\begin{array}{c}\text { Strand } \\
\text { wideh } \\
(\mathrm{mm})\end{array}$ & $\begin{array}{c}\text { Applied } \\
\text { load } \\
(\mathrm{kN})\end{array}$ & $\begin{array}{c}\text { Processing } \\
\text { temperature } \\
\left({ }^{\circ} \mathrm{C}\right)\end{array}$ \\
$\begin{array}{c}\text { Large } \\
\text { strands }\end{array}$ & 25.4 & 6.35 & $1,5,10,20$ & 400 \\
$\begin{array}{c}\text { Medium } \\
\text { wide strands } \\
\begin{array}{c}\text { Medium } \\
\text { slender } \\
\text { strands }\end{array}\end{array}$ & 12.7 & 6.35 & $1,5,10,20$ & 400 \\
$\begin{array}{c}\text { Small } \\
\text { strands }\end{array}$ & 12.7 & 3.18 & $1,5,10,20$ & 400 \\
UD & 6.35 & 3.18 & $1,5,10,20$ & 400 \\
\hline
\end{tabular}


Table 1-Model input parameters

\begin{tabular}{ccc}
\hline $\begin{array}{c}\text { Parameter name } \\
\text { (units) }\end{array}$ & Symbol & $\begin{array}{c}\text { Value } \\
\text { used }\end{array}$ \\
\hline Sample width $(\mathrm{m})$ & $W$ & 0.05 \\
Initial sample length $(\mathrm{m})$ & $L_{0}$ & 0.05 \\
Initial sample height $(\mathrm{m})$ & $h_{0}$ & 0.006 \\
Applied force $(\mathrm{N})$ & $F$ & $1000,2500,5000$ \\
Time step $(\mathrm{s})$ & $d t$ & 0.1 \\
Discretization size in $\mathrm{x}(\mathrm{m})$ & $d x$ & 0.000375 \\
Discretization size in $\mathrm{y}(\mathrm{m})$ & $d y$ & 0.0025 \\
\hline Zero shear rate viscosity & $7 ?_{0}$ & $7.45 \mathrm{e} 5$ \\
(Pa-s) & 7 & 52.2 \\
Relax ation time constant $(\mathrm{s})$ & $n$ & 0.59 \\
Shear-thinning ex ponent & $n$
\end{tabular}

